\title{
Aplicação da Cromatografia a Líquido em Substituição à Técnica de Radioimunoensaio como Auxílio Diagnóstico Visando ao Gerenciamento de Resíduos de Serviço da Saúde em Laboratório de Pesquisa
}

original

LUCIANE M. RIBEIRO Neto

EDUARDO K. SUGAWARA

IEDA T. N. VERRESCHI

Laboratório de Esteróides do

Departamento de Medicina

da Universidade Federal

de São Paulo (Unifesp),

São Paulo, SP, Brasil.

Recebido em 16/10/2007

Aceito em 19/8/2008
RESUMO

A elaboração do Plano de Gerenciamento dos Resíduos dos Serviços de Saúde, normatizado na RDC no $306 / 2004$, é de responsabilidade de todos os geradores. Sendo o radioimunoensaio (RIE) uma das técnicas mais empregadas, estudou-se o impacto da substituição dessa técnica por cromatografia líquida (HPLC), no aspecto da redução na geração de resíduos radioativos na rotina do Laboratório de Esteróides da Universidade Federal de São Paulo (Unifesp). Os resíduos gerados nas determinações de cortisol e $17 \alpha$-hidroxiprogesterona séricos foram classificados, e aqueles pertencentes aos grupos $\mathrm{B}$ e $\mathrm{C}$ foram avaliados. Observou-se que no emprego de RIE há geração de resíduos químicos (grupo $B$ ) e rejeitos radioativos (grupo C), enquanto no HPLC gerou-se apenas resíduos químicos. A adequação dessas técnicas teve vantagem de redução significativa no tempo de análise e, sobretudo, a eliminação e/ou a diminuição na geração de rejeitos radioativos, estimulando a sua aplicação para outras metodologias, bem como a implantação em outras unidades de pesquisas. (Arq Bras Endocrinol Metab 2008; 52/7:1172-1175)

Descritores: Gerenciamento de Resíduos; Cortisol; $17 \alpha$-hidroxiprogesterona; Radioimunoensaio; HPLC

\section{ABSTRACT}

Application of Liquid Chromatography in Substitution of the Radioimmunoassay Technique in Order to Reduce Residues Generated in Health Services in Research Laboratory.

Designing a Health Care Service Waste Management Plan, according to the RDC 306 rules, is a responsibility of all those who produce such waste. Since radioimmunoassay (RIA) is one of the most employed techniques, we studied the impact of replacing this technique by liquid chromatography (HPLC) with regard to the reduction of the radioactive residues routinely produced by the Unifesp steroid laboratory. The residues produced by the determination of serum cortisol and $17 \alpha$-hydroxyprogesterone were classified, and those belonging to groups $B$ and $C$ were evaluated. We observed that, when RIA is used, chemical residues (group B) and radioactive waste (group C) are produced, whereas HPLC generates only chemical residues. Adequation of these techniques showed to be advantageous, by significantly reducing the time of analysis and mainly by eliminating and/or reducing the generation of radioactive waste, encouraging its application to other methodologies, as well as its adoption by other research units. (Arq Bras Endocrinol Metab 2008; 52/7:1172-1175)

Keywords: Waste management; Cortisol; 17 $\alpha$-hydroxyprogesterone; Radioimmunoassay; HPLC 


\section{INTRODUÇÃO}

E m virtude do potencial de periculosidade dos resíduos gerados nos estabelecimentos de saúde, foi adotada a Resolução da Diretoria Colegiada (RDC) $n^{\circ}$ 306, de 7 de dezembro de 2004 (1), que dispõe sobre o regulamento técnico para o gerenciamento de resíduos de serviços de saúde. Esta resolução foi elaborada a partir do aprimoramento, da atualização e da complementação dos procedimentos contidos na resolução $\mathrm{RDC} \mathrm{n}^{\circ} 33$, de 25 de fevereiro de 2003 (2). A elaboração do Plano de Gerenciamento dos Resíduos dos Serviços de Saúde (PGRSS) é de responsabilidade de todos os geradores de resíduos de serviços da saúde (RSS), e está normatizado por meio da RDC n ${ }^{\circ} 306$ (1). Definem-se como geradores de RSS todos os serviços relacionados com o atendimento à saúde humana ou animal, inclusive estabelecimentos de ensino e pesquisa.

O primeiro passo para a implantação do PGRSS é o reconhecimento dos resíduos gerados. Os RSS são classificados pela RDC n ${ }^{\circ} 306 / 2004$ (1) em cinco grupos, a saber:

Grupo A - resíduos com a possível presença de agentes biológicos que, por suas características, podem apresentar riscos de infecção;

Grupo B - resíduos contendo substâncias químicas que podem apresentar risco à saúde pública ou ao meio ambiente, dependendo de suas características de inflamabilidade, corrosividade, reatividade e toxicidade;

Grupo C - quaisquer materiais resultantes de atividades humanas que contenham radionuclídeos em quantidades superiores aos limites de isenção especificados nas normas do Comissão Nacional de Energia Nuclear (CNEN) e para os quais a reutilização seja imprópria ou não prevista;

Grupo D - resíduos que não apresentem riscos biológicos, químicos ou radiológicos à saúde ou ao meio ambiente, podendo ser equiparados aos resíduos domiciliares; e

Grupo E - materiais perfurocortantes ou escarificantes.

O PGRSS além de gerenciar os resíduos e seus aspectos intra e extra-estabelecimentos, desde a geração até a disposição final, visa, também, a minimizar a produção de resíduos, objetivando, assim, a proteção dos trabalhadores e a preservação da saúde pública, dos recursos naturais e do meio ambiente.

Com o objetivo de atender o preconizado pela RDC n 306/2004 (1), o Laboratório de Esteróides da Unifesp tem investido na mudança metodológica das suas técnicas analíticas. Na rotina laboratorial de diagnósticos de patologias adrenais, destacam-se as determinações de cortisol e $17 \alpha$-hidroxiprogesterona séricos. O radioimunoensaio (RIE), uma das técnicas primordiais mais empregadas para estas determinações, que ainda tem ampla aplicação na rotina laboratorial, foi estudado quanto à sua substituição por cromatografia líquida de alta eficiência (HPLC), no aspecto da redução da geração de rejeitos radioativos (grupo C).

\section{MÉTODOS}

Os métodos avaliados empregados nas dosagens de cortisol e $17 \alpha$-hidroxiprogesterona séricos por RIE foram os rotineiramente utilizados no Laboratório de Esteróides da Unifesp $(3,4)$, técnica de competição em que o antígeno a ser medido compete com a quantidade limitada de antígeno marcado com substância radioativa $\left({ }^{3} \mathrm{H}\right.$ - trício) pelo sítio de ligação, o anticorpo. Estas determinações dispensam prévio tratamento da amostra. As metodologias empregadas na determinação por HPLC estão sendo utilizadas em substituição ao RIE e têm como base a separação cromatográfica em fase reversa precedidas por extração líquido-líquido empregando éter $(5,6)$, ambos aprovados pelo Comitê de Ética em Pesquisa da Unifesp. Na determinação do cortisol, a fase móvel empregada foi água-acetonitrila $(72: 28 ; \mathrm{v} / \mathrm{v})$, enquanto para a $17 \alpha$-hidroxiprogesterona a fase móvel foi água-metanol $(40: 60 ; \mathrm{v} / \mathrm{v})$. O tempo de análise cromatográfica foi de 15 e 14 minutos, respectivamente.

Os resíduos gerados na determinação de cortisol e $17 \alpha$-hidroxiprogesterona séricos, por RIE e por HPLC, foram classificados de acordo com a RDC n ${ }^{\circ} 306 / 2004$ (1), e apenas aqueles classificados como pertencentes aos grupos B e C foram avaliados. Para efeito de estudos comparativos tomou-se como base uma rotina equivalente a 100 amostras.

\section{RESULTADOS}

A classificação dos RSS gerados nas dosagens de cortisol e $17 \alpha$-hidroxiprogesterona séricos, respectivamente, empregando-se RIE e HPLC, bem como a descrição destes resíduos com as respectivas quantidades geradas na determinação de 100 amostras nas técnicas avaliadas estão reunidas na Tabela 1. 


\section{DIscussão}

Comparando-se os RSS gerados nas dosagens por RIE e por HPLC, na técnica de RIE há geração de resíduos químicos (grupo B) e rejeitos radioativos (grupo C), enquanto na técnica de HPLC geram-se apenas resíduos químicos. Os rejeitos do grupo $\mathrm{C}$ gerados nas dosagens por RIE estão nas formas líquida e sólida. Estes RSS necessitam de armazenamento temporário por tempo relativamente extenso em função da meia-vida $\left(\mathrm{t}_{1 / 2}\right)$ física do radioisótopo trício- ${ }^{3} \mathrm{H}$ (12,3 anos). Após respeitadas as normas preestabelecidas para este radioisótopo, ou seja, concentração radioativa do rejeito líquido com valor abaixo do limite de eliminação $(3,7 \times$ $\left.10^{9} \mathrm{~Bq} / \mathrm{m}^{3}\right)(7,8)$, os rejeitos líquidos são reclassificados como resíduos pertencentes ao grupo B e, então, encaminhados para destinação final. Somente os rejeitos sólidos gerados na primeira fase do ensaio radioativo devem ser encaminhados a tratamento final ao Instituto de Pesquisa e Energia Nuclear (Ipen). Os demais rejeitos sólidos que estão abaixo do limite de eliminação, $75 \mathrm{~Bq} / \mathrm{g}$, são reclassificados como resíduos pertencentes ao grupo A e, então, descartados no sistema de coleta de resíduo hospitalar $(1,7)$.
Embora o volume de resíduo químico gerado por HPLC seja superior ao gerado por RIE, na cromatografia a líquido exclui-se a geração de resíduos do grupo $\mathrm{C}$, bem como a geração de resíduos sólidos.

A substituição da técnica do RIE pela de HPLC na determinação de cortisol sérico pode ser aplicada à rotina laboratorial; já na determinação da $17 \alpha$-hidroxiprogesterona sérica a substituição está limitada a concentrações elevadas desse esteróide. Os limites de quantificação de cortisol e $17 \alpha$-hidroxiprogesterona séricos na metodologia analítica desenvolvida empregando HPLC foram, respectivamente, $2,5 \mu \mathrm{g} / \mathrm{dL}$ e $500 \mathrm{ng} / \mathrm{dL}$. De fato, o emprego da técnica de HPLC para a $17 \alpha$-hidroxiprogesterona objetivou o diagnóstico da hiperplasia adrenal congênita da forma clássica, com significativa redução do tempo de análise (6).

Por outro lado, evidenciou-se a vantagem adicional da redução em $22 \%$ dos rejeitos radioativos gerados nos 24 meses desde a introdução desta metodologia neste laboratório.

A adequação das técnicas empregadas para o diagnóstico de patologias adrenais teve vantagem de redução significativa no tempo de análise e, sobretudo, a eliminação e/ou a diminuição na geração de rejeitos radioati-

Tabela 1. Resíduos de serviço da saúde (RSS) gerados na dosagem de cortisol e 17 $\alpha$-hidroxiprogesterona séricos por radioimunoensaio (RIE) e por cromatografia a líquido (HPLC) para a determinação de 100 amostras.

\begin{tabular}{|c|c|c|c|c|}
\hline & \multicolumn{2}{|c|}{ Cortisol } & \multicolumn{2}{|c|}{$17 \alpha$-hidroxiprogesterona } \\
\hline & RIE & HPLC & RIE & HPLC \\
\hline RSS grupo B & $\begin{array}{c}980 \mathrm{~mL} \text { de solução } \\
\text { tampão pH = 7,0 } \\
\text { contendo 1\% de metanol } \\
580 \mathrm{~mL} \text { de solução* (com } \\
\text { concentração radioativa } \\
\text { com valor abaixo do } \\
\text { limite de eliminação) }\end{array}$ & $\begin{array}{c}1.700 \mathrm{~mL} \text { de solução } \\
\text { aquosa de acetonitrila a } \\
33 \%\end{array}$ & $\begin{array}{c}100 \text { mL de solução } \\
\text { tampão pH = 7,0 } \\
580 \text { mL de solução* (com } \\
\text { concentração radioativa } \\
\text { com valor abaixo do } \\
\text { limite de eliminação) }\end{array}$ & $\begin{array}{c}1.700 \mathrm{~mL} \text { de solução } \\
\text { aquosa de metanol } \\
\text { a } 72 \%\end{array}$ \\
\hline RSS grupo C & $\begin{array}{c}580 \mathrm{~mL} \text { de solução } \\
\text { radioativa }\left({ }^{3} \mathrm{H}\right)^{*} \\
0,25 \mathrm{~g} \text { de carvão ativado } \\
200 \text { tubos de } \\
\text { polipropileno } \\
200 \text { tubos tipo KMA }\end{array}$ & Não gerado & $\begin{array}{c}580 \mathrm{~mL} \text { de solução } \\
\text { radioativa }(3 \mathrm{H})^{*} \\
0,25 \mathrm{~g} \text { de carvão ativado } \\
200 \text { tubos de } \\
\text { polipropileno } \\
200 \text { tubos tipo KMA }\end{array}$ & Não gerado \\
\hline
\end{tabular}

* Solução tampão $\left(\mathrm{Na}_{2} \mathrm{HPO}_{4} / \mathrm{NaH}_{2} \mathrm{PO}_{4}\right)$ e líquido de cintilação (nonylfenolethoxylate). 
vos, garantindo a proteção aos trabalhadores em relação a possíveis contaminações durante eventual manuseio inadequado do material radioativo e dos rejeitos gerados e preservação da saúde pública e do meio ambiente. $\mathrm{O}$ alto investimento no equipamento HPLC visando ao aprimoramento analítico, no caso das dosagens de cortisol e $17 \alpha$-hidroxiprogesterona séricos, estimula sua otimização, permitindo diversificações metodológicas. A aplicação do HPLC em laboratórios de apoio à pesquisa e de diagnóstico está em ascensão em razão da gama de diversificações analíticas, ao contrário dos equipamentos para contagem de radioatividade utilizados na técnica de RIE cuja aplicação é restrita. No início da atividade de um laboratório, o custo com equipamentos, treinamento, qualificação profissional e implantação de sistema de gestão, quer seja de qualidade, quer seja de resíduos, os custos se equiparam para ambas as técnicas abordadas nesse trabalho.

A implantação do PGRSS é de relevante importância em laboratórios acadêmicos, embora sejam notórias as dificuldades de implantação de tal conceito, ao contrário de laboratórios de rede privada em que a gestão de geração de resíduos é incorporada à rotina laboratorial, tendo em vista o cumprimento das legislações e das certificações. Os presentes resultados devem incentivar a sua aplicação para outras metodologias, bem como a implantação de medidas para gerir resíduos de serviços da saúde em outras unidades de pesquisas.

Agradecimentos. Ao Conselho Nacional de Desenvolvimento Científico e Tecnológico (CNPq) ( $\left.\mathrm{n}^{\circ} 478243 / 2004-9\right)$ pelo apoio financeiro ao desenvolvimento da metodologia para a determinação do cortisol sérico por HPLC. À Maria Fernanda Salgado Santos Mattos, engenheira no Núcleo de Proteção Radiológica do Departamento de Diagnóstico por Imagem da Unifesp, pela colaboração nas informações referentes aos rejeitos radioativos. Os autores declaram não haver conflitos de interesse científico neste artigo.

\section{REFERÊNCIAS}

1. Anvisa. Agência Nacional de Vigilância Sanitária. Resolução 306, de 7 de dezembro de 2004. Disponível em: http://www. anvisa.gov.br.

2. Anvisa. Agência Nacional de Vigilância Sanitária. Resolução 33, de 25 de fevereiro de 2003. Disponível em: http//www. anvisa.gov.br.

3. Vieira JGH, Russo EMK, Maciel RMB, Germek AO, Antunes LAN. Método radioimunológico para dosagem do cortisol sérico. Rev Bras Patol Clin. 1979;15(3):125-30.

4. Vieira JGH, Russo EMK, Maciel RMB, Germek AO, Verreschi ITN. Radioimumoensaio para $17 \alpha$-hidroxiprogesterona sérica: considerações metodológicas. Arq Bras Endocrinol Metab. 1980;24(1):24-30.

5. Sugawara EK, Ribeiro-Neto LM, Oliveira KC, Verreschi ITN Reduzindo geração de resíduo e o tempo de análise na determinação de cortisol sérico. Arq. Bras Endocrinol Metab. 2007; 51(3,S1):S87.

6. Sugawara EK, Ribeiro-Neto LM, Fernandes VFT, Kater CE, Verreschi ITN. Aplicação da cromatografia líquida de alta eficiência para quantificação direta da $17 \alpha$-hidroxiprogesterona sérica. Rev Bras Cien Farm. 2004;40(3):327-33.

7. Comissão Nacional de Energia Nuclear. Gerência de rejeitos radioativos em instalações radiativas. Rio de Janeiro: CNEN, 1985. CNEN-NE-6.05.

8. Companhia de Tecnologia de Saneamento Básico. Gerenciamento de resíduos químicos provenientes de estabelecimentos de serviços de saúde (Procedimento). São Paulo: CETESB 2003. Cetesb-P4.262.

Endereço para correspondência:

leda T. N. Verreschi

Lab. Esteróides, Disc. Endocrinologia - EPM-Unifesp

Rua Pedro de Toledo, 781, $13^{\circ}$ andar

040039-032 São Paulo, SP

E-mail: ieda.verreschi@unifesp.br 